\title{
Estudo comparativo da avifauna em áreas verdes urbanas da região metropolitana de Porto Alegre, sul do Brasil
}

\author{
Marcelo Fischer Barcellos dos Santos* \\ Cristina Vargas Cademartori \\ Grupo de Pesquisa Meio Ambiente e Impacto Antrópico \\ Pós-Graduação stricto sensu e Pesquisa, Centro Universitário La Salle \\ Av. Victor Barreto, 2288, CEP 92010-000, Canoas - RS, Brasil \\ *Autor para correspondência \\ fischeri_80@hotmail.com
}

Submetido em 25/04/2009

Aceito para publicação em 12/01/2010

\section{Resumo}

Este estudo teve como objetivo comparar a composição e a riqueza de espécies de aves em áreas verdes da região metropolitana de Porto Alegre. Compilaram-se dados obtidos da literatura quanto à composição de espécies de aves em 14 áreas verdes. Realizou-se análise de similaridade (Jaccard) com o propósito de comparar a composição de espécies entre distintas áreas verdes. Utilizou-se análise de regressão múltipla, aplicando-se o método stepwise, para testar se a riqueza de espécies de aves é influenciada pelas seguintes características das áreas verdes urbanas: dimensões, variedade de hábitats, área e distância do vizinho mais próximo. Compilou-se um total de 224 espécies de aves para as áreas verdes consultadas. A análise de Jaccard demonstrou que áreas de maiores dimensões apresentaram composição similar entre si e distinta de áreas de menores dimensões. O teste de regressão múltipla indicou que houve correlação positiva entre a riqueza de espécies e os fatores estudados, exceto pela distância entre as áreas. Concluiu-se que áreas verdes de maiores dimensões e com maior heterogeneidade de hábitats podem sustentar maior riqueza de espécies de aves em ambientes urbanizados. Entretanto, a importância de áreas pequenas não deve ser desprezada, especialmente nos casos em que se constituem nos últimos ou principais remanescentes de determinadas espécies.

Unitermos: aves urbanas, áreas urbanas, região metropolitana de Porto Alegre

\section{Abstract}

Comparative study of bird communities in green areas of the Porto Alegre metropolitan region, South of Brazil. Our goal was to compare the bird richness and composition among different urban green areas in the metropolitan region of Porto Alegre. Data on the composition of species in 14 green areas were collected from the literature. Similarity analysis (Jaccard's) was used to compare the areas in relation to bird composition. We used multiple regression analysis in addition to the stepwise method in order to establish whether bird richness in the urban green areas was influenced by the following factors: dimension, variety of habitats, area and distance of the nearest neighbor. Our data compilation registered 224 bird species in the consulted areas. Jaccard's analysis showed that larger green areas are more similar in their composition of bird species than smaller ones. Multiple regression analysis demonstrated positive correlations between bird richness and the analyzed factors (except for distance between areas). We concluded that, despite the importance of small green areas to certain bird species, larger ones with higher habitat heterogeneity are able to support significant bird richness, and that these areas are important to the maintenance of many bird species in urban areas.

Key words: Porto Alegre metropolitan region, urban avifauna, urban landscapes 


\section{Introdução}

O processo de urbanização produz alterações nos ambientes naturais, modificando substancialmente a paisagem. Entre as modificações, pode-se citar a fragmentação das áreas originais e a consequente formação de mosaicos de ilhas de diferentes formas e tamanhos, onde a vegetação nativa é, geralmente, substituída por espécies oportunistas ou exóticas (Matarazzo-Neuberguer, 1995; Gimenes e Anjos, 2003). Tais alterações podem exercer grande influência sobre as comunidades de aves em longo prazo (Anjos e Laroca, 1989; Anjos, 1990; Grillo e Bencke, 1995; Sick, 1997; Accordi, 2001; Fontana, 2005), tornando a composição da avifauna em ambientes urbanos, altamente degradados, bastante distinta de ambientes preservados onde ainda existam elementos primários de vegetação. Assim, a avifauna encontrada em grandes centros urbanos, na maioria das vezes, é composta de espécies mais generalistas quanto à escolha de hábitats e, em vários casos, algumas se beneficiam dos processos de urbanização (Willis, 2000; Accordi, 2001; Fontana, 2005). Alguns estudos demonstraram que a riqueza de espécies de aves em ambientes urbanos está relacionada com as diferentes fitofisiomias encontradas, que acabam por propiciar hábitats para muitas aves (Krügel e Anjos, 2000; Lopes e Anjos, 2006; Valadão et al., 2006).

No Rio Grande do Sul, há uma série de estudos relativos à composição de espécies de aves em áreas verdes urbanas, principalmente na região metropolitana de Porto Alegre, Voss (1976; 1977a; 1977b; 1977c; 1979; 1981), Voss e Sander (1979a; 1979b), Sander e Voss (1982), Tampson (1990), Grillo e Bencke (1995), Mendonça-Lima e Fontana (2000), Accordi (2001), Efe et al. (2001) e Fontana (2005). Embora, em sua maioria, se restrinjam a listas de aves constatadas em cada área de estudo, tais estudos foram importantes para o conhecimento da ecologia de muitas das espécies registradas (Fontana, 2005). A partir de listas de espécies, é possível realizar comparações entre as comunidades de aves de diferentes áreas e correlacionar dados de ocorrência com aspectos fitofisionômicos de cada local. A despeito da relevância dessas contribuições para o conhecimento sobre a diversidade e ecologia das aves, nenhuma delas testou a influência de aspectos físicos das áreas sobre a riqueza de espécies. O padrão espacial dos ecossistemas urbanos pode constituir uma explicação para a variação na riqueza de espécies de aves registradas em áreas verdes metropolitanas, uma vez que edificações e outras construções antrópicas podem representar barreiras à dispersão de espécies (Accordi, 2001).

Este trabalho reúne informações provenientes de estudos publicados sobre a riqueza e composição de espécies de aves na região metropolitana de Porto Alegre. O principal objetivo foi produzir uma lista geral de espécies para a região, identificando seus padrões de distribuição. Verificou-se, também, se o tamanho da área, o número de hábitats, área e distância do vizinho mais próximo influenciam a riqueza de espécies de aves na região.

\section{Material e Métodos}

\section{Área de estudo}

A região metropolitana de Porto Alegre, localizada no extremo leste da Depressão Central do Rio Grande do Sul, é formada por 31 municípios, comportando cerca de $37 \%$ da população do Rio Grande do Sul e constituindo a área mais densamente povoada do estado (Secretaria de Planejamento e Gestão, 2008). A paisagem consiste em um complexo mosaico ambiental que integra construções urbanas, áreas verdes, arrozais, áreas úmidas e campos. O clima na região é do tipo $\mathrm{Cfa}$ na classificação de Köppen, com precipitação média de $1600 \mathrm{~mm}$ e temperatura média, no verão, de $21^{\circ} \mathrm{C}$, e no inverno, de $12^{\circ} \mathrm{C}$ (Datasinos, 2006).

\section{Métodos}

\section{Revisão da literatura e seleção das áreas}

Listas de espécies de aves registradas em 14 áreas verdes da região metropolitana de Porto Alegre, disponíveis na literatura científica, foram consultadas (Tabela 1, Figura 1). Consideraram-se áreas verdes, neste estudo, locais com vegetação nativa ou exótica, mas estritamente de uso público, tais como praças, 
TABELA 1: Fontes utilizadas para comparação da avifauna em manchas verdes urbanas da região metropolitana de Porto Alegre, em que constam listas de espécies de aves, e informações sobre as áreas de estudo. 1 - Edificações urbanas (prédios, casas, quadras de esporte, etc); 2 - Capão nativo (agrupamento arbóreo de vegetação nativa); 3 - Lago (vegetação palustre associada); 4 - Área aberta (campos ou áreas abertas com vegetação rasteira associada e árvores esparsas); 5 - Bosque (árvores e arvoretas de menor porte, podendo ser de vegetação nativa ou exótica); 6 - Eucaliptal (plantio de Eucalyptus sp. sem fins comerciais); 7 - Pomar (plantação de árvores frutíferas); 8 - Banhado (área úmida com vegetação palustre associada); 9 - Arroio (curso d'água); 10 - Vegetação exótica (presença de vegetação exótica ornamental); 11 - Maricazal (vegetação predominante de Mimosa bimucronata).

\begin{tabular}{|c|c|c|c|c|}
\hline Fonte & Local & Município & $\begin{array}{c}\text { Área } \\
\text { (ha) }\end{array}$ & Hábitats \\
\hline Tampson (1990) & Morro do Espelho & São Leopoldo & 19 & $1,2,4,5,7$ \\
\hline Grillo e Bencke $(1995)^{\#}$ & UNISINOS & São Leopoldo & 80 & $1,2,3,4,5,6$ \\
\hline Voss (1977c) & Zoológico de Sapucaia do Sul & Sapucaia do Sul & 120 & $1,2,3,4,5,6$ \\
\hline Accordi (2001) & Parque Municipal Getúlio Vargas & Canoas & $195^{*}$ & $1,2,3,4,5,6$ \\
\hline Oliveira (2006) & Campus UNILASALLE & Canoas & 5 & 1,5 \\
\hline Efe et al. (2001) & Parque Marinha do Brasil & Porto Alegre & 70,7 & $1,2,3,11$ \\
\hline Efe et al. (2001) & $\begin{array}{c}\text { Parque Maurício Sirotsky Sobri- } \\
\text { nho }\end{array}$ & Porto Alegre & 65 & $1,2,4$ \\
\hline Mendonça-Lima e Fontana (2000) & Porto Alegre Country Clube & Porto Alegre & 47 & $1,2,3,4,9,10$ \\
\hline $\begin{array}{l}\text { Voss (1981), Fundação Zoobotânica do } \\
\text { Rio Grande do Sul (2004) }\end{array}$ & Jardim Botânico de Porto Alegre & Porto Alegre & 43 & $1,2,3,4,11$ \\
\hline Sander e Voss (1982), Efe et al. (2001) & Parque Farroupilha & Porto Alegre & 37,5 & $1,2,3,5$ \\
\hline Efe et al. (2001) & Parque Chico Mendes & Porto Alegre & 25,2 & $1,5,6$ \\
\hline Efe et al. (2001) & Parque Mascarenhas de Moraes & Porto Alegre & 18,3 & $1,2,8$ \\
\hline Efe et al. (2001) & Parque Moinhos de Vento & Porto Alegre & 11,5 & $1,3,5$ \\
\hline Efe et al. (2001) & Parque Gabriel Knijnik & Porto Alegre & 12 & $1,2,6,7,8$ \\
\hline
\end{tabular}

\# dados acumulados de Voss e Sander (1979) e Lucchese e Petry (não publicado); * área total amostrada por Accordi (2001).

parques e campus de universidades. Áreas protegidas, onde o acesso de pessoas é limitado ou restrito, foram excluídas da investigação. Da mesma forma, o aeroporto Salgado Filho, uma vez que a comunidade de aves desta área sofre a influência de uma série de impactos decorrentes do trânsito e sons emitidos pelas aeronaves. Os três sítios contíguos estudados por Accordi (2001) no município de Canoas foram considerados como uma única área verde.

\section{Análises}

Utilizou-se o teste de regressão múltipla (empregando-se o modelo stepwise) para verificar se o tamanho, heterogeneidade de hábitats, área e distância do vizinho mais próximo (área verde mais próxima consultada) influenciam a riqueza de espécies de aves nas áreas verdes (Tabela 1). Tais informações foram extraídas dos trabalhos consultados ou estavam disponíveis em SMAM (2009). O teste foi executado no programa Systat versão 11 (Wilkinson, 2004), sendo que os resultados foram considerados significativos para $\mathrm{P}<0,05$.

O coeficiente de Jaccard foi empregado, por meio do programa MVSP (Kovach, 2005), para investigar a similaridade na composição de espécies de aves entre as áreas estudadas utilizando o método de ligação média não ponderada (UPGMA). O gráfico resultante desta análise gera um dendograma com valores variando de 0 a 1 , sendo que quanto mais próximo de 1 mais similares são os grupos formados quanto à composição de espécies de aves.

Elaborou-se uma lista de ocorrência de aves em áreas verdes urbanas, registrando-se a presença ou a ausência de espécies nas referidas áreas, com o objetivo 


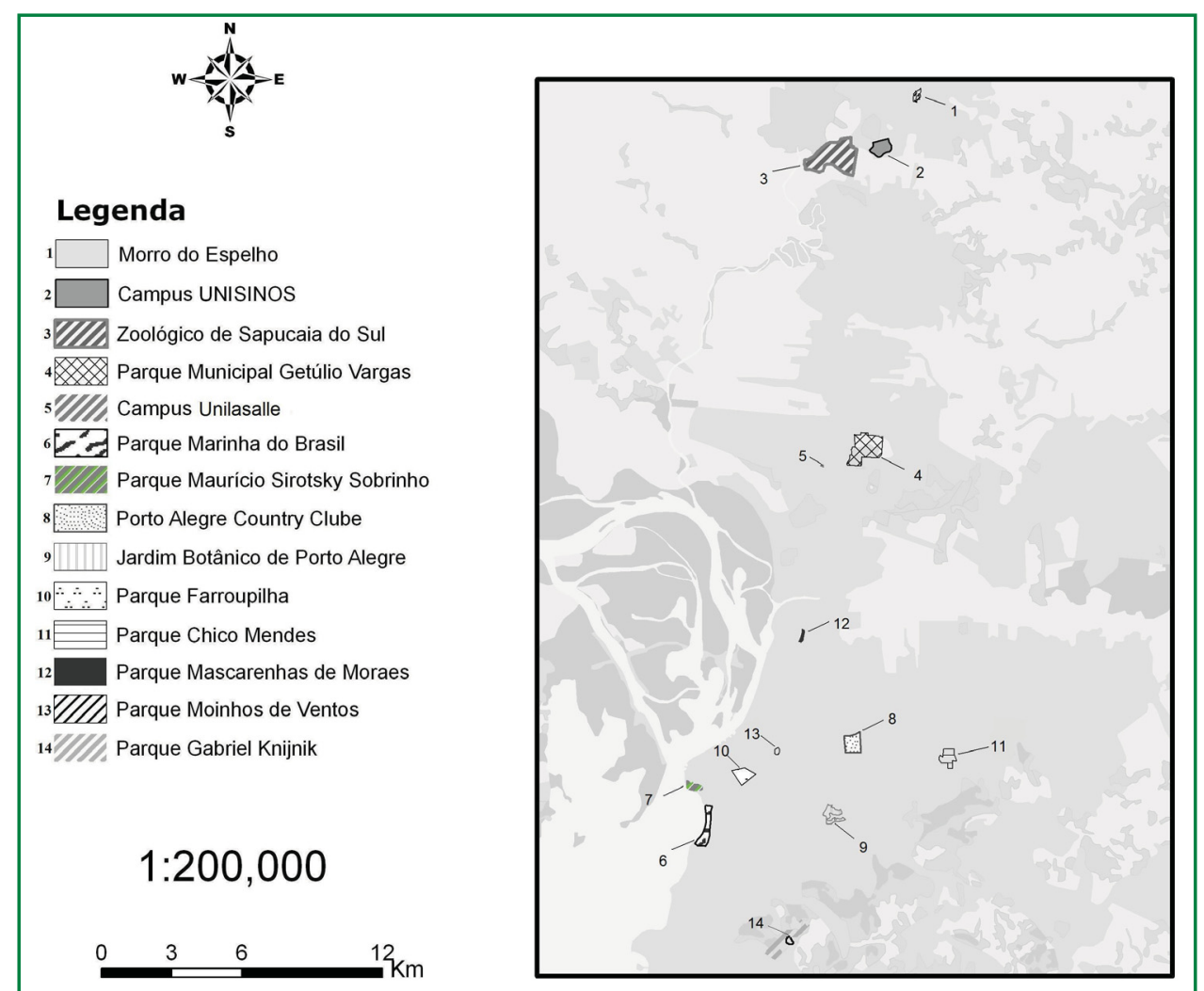

FIGURA 1. Localização das áreas verdes consultadas na região metropolitana de Porto Alegre, Rio Grande do Sul, Brasil.

de verificar a distribuição das espécies nas áreas verdes da região metropolitana de Porto Alegre. Este parâmetro foi obtido através da razão entre o número de registros de cada espécie em determinada área e o número total de áreas analisadas. Adotaram-se, a partir dos resultados obtidos, três categorias de distribuição local de espécies: espécies com distribuição restrita (encontradas em até quatro áreas verdes ou 28,5\%), espécies parcialmente distribuídas (registradas em cinco a sete áreas verdes ou $35,7-57,1 \%$ ) e amplamente distribuídas (encontradas em mais de sete áreas verdes ou acima de 64,2\%). Aves introduzidas em algumas áreas, como Cygnus melancoryphus, Cairina moschata, psitacídeos exóticos a região metropolitana de Porto alegre, bem como táxons que não foram identificados em nível específico nos trabalhos consultados não foram incluídos nas análises. O registro de Megascops atricapilla, realizado por Voss (1977c) no Zoológico de Sapucaia do Sul, foi tido como Megascops sanctaecatarinae, uma vez que, de acordo com Belton (1994), M. atricapilla ainda não foi constatada no estado nem consta como espécie ocorrente no Rio Grande do Sul (Bencke, 2001). A ordem sistemática adotada segue o Comitê Brasileiro de Registros Ornitológicos (2008).

\section{Resultados e Discussão}

\section{Composição específica}

Um total de 224 espécies de aves foi compilado para as 14 áreas verdes da região metropolitana de Porto Alegre (Tabela 2), correspondendo a aproximadamente $36 \%$ das espécies registradas para o estado do Rio Grande do Sul (Bencke, 2001). Este número pode ser considerado elevado, uma vez que ambientes antropizados são hostis a uma grande parcela da avifauna (Fontana, 2005). 
TABELA 2: Espécies de aves registradas em 14 áreas verdes da região metropolitana de Porto Alegre, sul do Brasil. (1) Morro do Espelho (Tampson, 1990), (2) Campus da Universidade do Vale do Rio dos Sinos, UNISINOS (Grillo e Bencke, 1995), (3) Parque Zoológico de Sapucaia do Sul (Voss, 1977c), (4) Parque Municipal Getúlio Vargas (Accordi, 2001), (5) Centro Universitário La Salle (Oliveira, 2006), (6) Parque Marinha do Brasil (Efe et al., 2001), (7) Parque Maurício Sirotski Sobrinho (Efe et al., 2001), (8) Porto Alegre Country Clube (MendonçaLima e Fontana, 2000), (9) Jardim Botânico de Porto Alegre (Voss 1981, Fundação Zoobotânica do Rio Grande do Sul, 2004), (10) Parque Farroupilha (Sander e Voss, 1982; Efe et al., 2001), (11) Parque Chico Mendes (Efe et al. 2001), (12) Parque Mascarenhas de Moraes (Efe et al., 2001), (13) Parque Moinhos de Vento (Efe et al., 2001) e (14) Parque Gabriel Knijnik (Efe et al., 2001). "D” distribuição das espécies de aves considerando-se as áreas analisadas.

\begin{tabular}{|c|c|c|c|c|c|c|c|c|c|c|c|c|c|c|c|}
\hline \multirow{2}{*}{ Família/Espécie } & \multicolumn{14}{|c|}{ Áreas Verdes } & \multirow{2}{*}{ D } \\
\hline & 1 & 2 & 3 & 4 & 5 & 6 & 7 & 8 & 9 & 10 & 11 & 12 & 13 & 14 & \\
\hline \multicolumn{16}{|l|}{ Tinamidae } \\
\hline Crypturellus obsoletus & & & & & & & & & & & & & & $\mathrm{x}$ & 7,14 \\
\hline Nothura maculosa & & $\mathrm{x}$ & & $\mathrm{x}$ & & & & & $\mathrm{x}$ & & & $\mathrm{x}$ & & & 28,57 \\
\hline \multicolumn{16}{|l|}{ Anatidae } \\
\hline Dendrocygna bicolor & & & $\mathrm{x}$ & & & & & & & & & & & & 7,14 \\
\hline Dendrocygna viduata & $\mathrm{x}$ & $\mathrm{x}$ & $\mathrm{x}$ & $\mathrm{x}$ & & & & & & & & $\mathrm{x}$ & & & 35,71 \\
\hline Sarkidiornis sylvicola & & & $\mathrm{x}$ & & & & & & & & & & & & 7,14 \\
\hline Amazonetta brasiliensis & & & $\mathrm{x}$ & $\mathrm{x}$ & & & & & & & & $\mathrm{x}$ & & & 21,43 \\
\hline Anas georgica & & & $\mathrm{x}$ & & & & & & & & & & & & 7,14 \\
\hline \multicolumn{16}{|l|}{ Cracidae } \\
\hline Ortalis guttata & & & & & & & & & & & & & & $\mathrm{x}$ & 7,14 \\
\hline \multicolumn{16}{|l|}{ Podicipedidae } \\
\hline Tachybaptus dominicus & & $\mathrm{x}$ & $\mathrm{x}$ & & & & & & & & & & & & 14,29 \\
\hline Podilymbus podiceps & & $\mathrm{x}$ & $\mathrm{x}$ & $\mathrm{x}$ & & & & & & & & & & & 21,43 \\
\hline \multicolumn{16}{|l|}{ Phalacrocoracidae } \\
\hline Phalacrocorax brasilianus & & $\mathrm{x}$ & & $\mathrm{x}$ & & $\mathrm{x}$ & $\mathrm{x}$ & & & $\mathrm{x}$ & & $\mathrm{x}$ & $\mathrm{x}$ & & 50 \\
\hline \multicolumn{16}{|l|}{ Anhingidae } \\
\hline Anhinga anhinga & & $\mathrm{x}$ & & & & & & & & & & & & & 7,14 \\
\hline \multicolumn{16}{|l|}{ Ardeidae } \\
\hline Ixobrychus involucris & & & & & & & & & $\mathrm{x}$ & & & $\mathrm{x}$ & & & 14,29 \\
\hline Nycticorax nycticorax & & $\mathrm{x}$ & $\mathrm{x}$ & $\mathrm{x}$ & & $\mathrm{x}$ & $\mathrm{x}$ & & $\mathrm{x}$ & $\mathrm{x}$ & $\mathrm{x}$ & $\mathrm{x}$ & $\mathrm{x}$ & & 71,43 \\
\hline Nyctanassa violacea & & & $\mathrm{x}$ & & & & & & & & & & & & 7,14 \\
\hline Butorides striata & $\mathrm{x}$ & $\mathrm{x}$ & $\mathrm{x}$ & $\mathrm{x}$ & & $\mathrm{x}$ & & $\mathrm{x}$ & $\mathrm{x}$ & & & $\mathrm{x}$ & & & 57,14 \\
\hline Bubulcus ibis & & $\mathrm{x}$ & & $\mathrm{x}$ & & & $\mathrm{x}$ & & & $\mathrm{x}$ & & $\mathrm{x}$ & & & 35,71 \\
\hline Ardea cocoi & & & & & & & $\mathrm{x}$ & & & & & & & & 7,14 \\
\hline Ardea alba & $\mathrm{x}$ & $\mathrm{x}$ & $\mathrm{x}$ & $\mathrm{x}$ & & $\mathrm{x}$ & $\mathrm{x}$ & $\mathrm{x}$ & $\mathrm{x}$ & $\mathrm{x}$ & $\mathrm{x}$ & $\mathrm{x}$ & $\mathrm{x}$ & & 85,71 \\
\hline Syrigma sibilatrix & & $\mathrm{x}$ & $\mathrm{x}$ & $\mathrm{x}$ & & $\mathrm{x}$ & $\mathrm{x}$ & $\mathrm{x}$ & $\mathrm{x}$ & & $\mathrm{x}$ & & & & 57,14 \\
\hline Egretta thula & $\mathrm{x}$ & $\mathrm{x}$ & $\mathrm{x}$ & $\mathrm{x}$ & & $\mathrm{x}$ & $\mathrm{x}$ & $\mathrm{x}$ & $\mathrm{x}$ & & & $\mathrm{x}$ & & & 64,29 \\
\hline \multicolumn{16}{|l|}{ Threskiornithidae } \\
\hline Plegadis chihi & & & & $\mathrm{x}$ & & $\mathrm{x}$ & $\mathrm{x}$ & & & & & $\mathrm{x}$ & $\mathrm{x}$ & & 35,71 \\
\hline Phimosus infuscatus & & $\mathrm{x}$ & $\mathrm{x}$ & $\mathrm{x}$ & & $\mathrm{x}$ & $\mathrm{x}$ & $\mathrm{x}$ & $\mathrm{x}$ & $\mathrm{x}$ & & $\mathrm{x}$ & & $\mathrm{x}$ & 71,43 \\
\hline Platalea ajaja & & & & & & & $\mathrm{x}$ & & & & & & & & 7,14 \\
\hline \multicolumn{16}{|l|}{ Cathartidae } \\
\hline Cathartes aura & & $\mathrm{X}$ & $\mathrm{x}$ & & $\mathrm{x}$ & & & & & & & $\mathrm{x}$ & & $\mathrm{x}$ & 35,71 \\
\hline
\end{tabular}


Coragyps atratus

$\begin{array}{lllllll}\mathrm{X} & \mathrm{x} & \mathrm{X} & \mathrm{X} & \mathrm{x} & \mathrm{X} & \mathrm{X}\end{array}$

\section{Accipitridae}

Elanus leucurus

Rostrhamus sociabilis

Ictinia plumbea

Circus buffoni

Accipiter striatus

Buteogallus urubitinga

Heterospizias meridionalis

Percnohierax leucorrhous

Rupornis magnirostris

Buteo albicaudatus

Buteo brachyurus

\section{Falconidae}

Caracara plancus

Milvago chimachima

Milvago chimango

Falco sparverius

Falco femoralis

Falco peregrinus

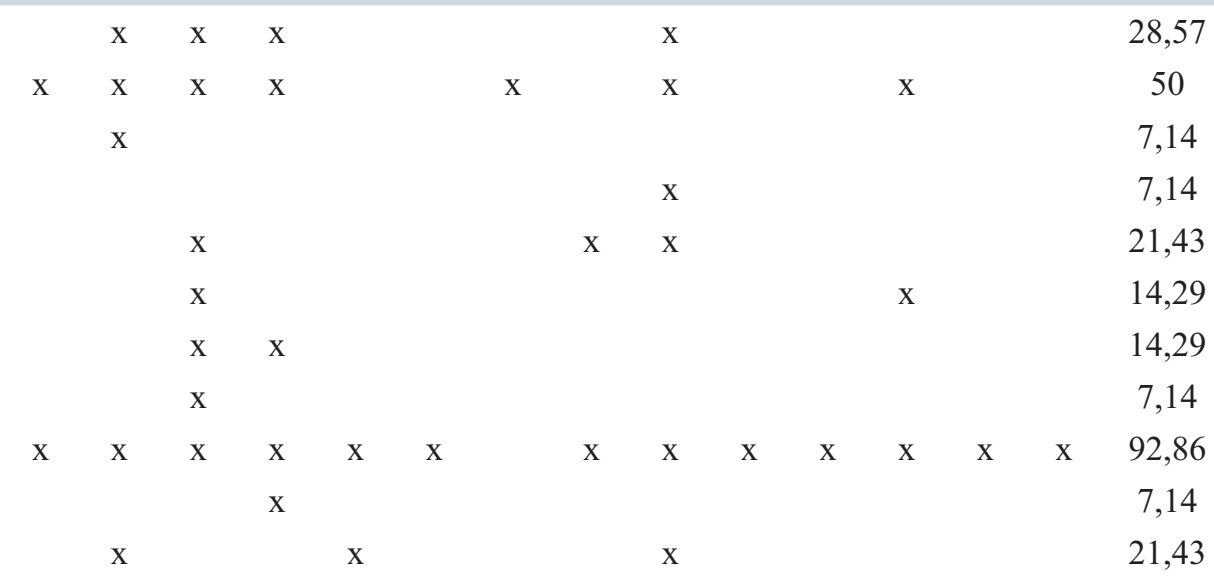

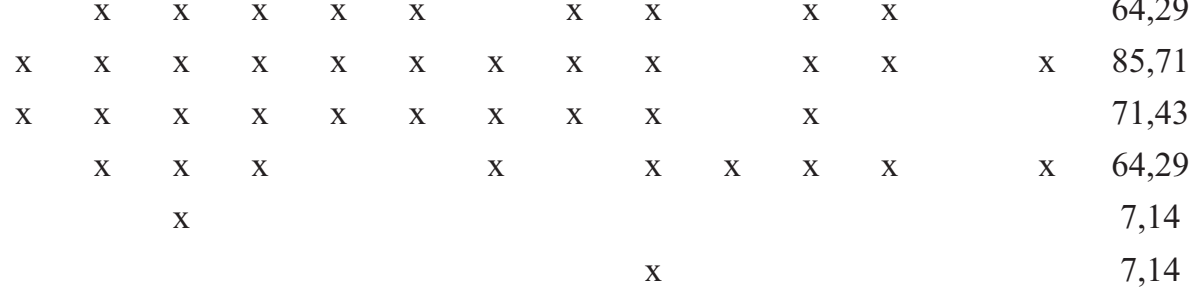

\section{Aramidae}

Aramus guarauna

$\mathrm{X}$

7,14

\section{Rallidae}

Aramides cajanea

$\mathrm{x}$

7,14

Aramides saracura

Laterallus melanophaius

Pardirallus nigricans

$\mathrm{X} \quad \mathrm{X} \quad \mathrm{X}$

$\begin{array}{lll}\mathrm{X} & \mathrm{X} & \mathrm{X}\end{array}$

$\mathrm{X}$

x $\quad 64,29$

$\mathrm{X}$

Pardirallus sanguinolentus

Gallinula chloropus

Gallinula melanops

X $\quad$ X $\quad$ X

X $\quad \mathrm{X}$

X

14,29

$\mathrm{x} \quad \mathrm{X}$

57,14

Porphyrio martinica

$\mathrm{x}$

14,29

14,29

\section{Charadriidae}

Vanellus chilensis

$\mathrm{x} \quad \mathrm{x}$

$\mathrm{X}$

$\mathrm{x}$

$\mathrm{x}$

\section{Recurvirostridae}

\begin{tabular}{|c|c|c|c|c|c|c|c|c|c|}
\hline Himantopus melanurus & & & $\mathrm{x}$ & $\mathrm{x}$ & $\mathrm{x}$ & & & $\mathrm{x}$ & 28,57 \\
\hline \multicolumn{10}{|l|}{ Scolopacidae } \\
\hline Gallinago paraguaiae & & & & $\mathrm{x}$ & & & $\mathrm{x}$ & $\mathrm{x}$ & 21,43 \\
\hline Tringa solitaria & & & $\mathrm{x}$ & & & & & & 7,14 \\
\hline Tringa melanoleuca & & & & & & & & $\mathrm{x}$ & 7,14 \\
\hline \multicolumn{10}{|l|}{ Jacanidae } \\
\hline Jacana jacana & $\mathrm{x}$ & $\mathrm{x}$ & $\mathrm{x}$ & $\mathrm{x}$ & $\mathrm{x}$ & $\mathrm{x}$ & $\mathrm{x}$ & $\mathrm{x}$ & 57,14 \\
\hline \multicolumn{10}{|l|}{ Laridae } \\
\hline Chroicocephalus maculipennis & & & $\mathrm{x}$ & & $\mathrm{x}$ & $\mathrm{x}$ & & & 21,43 \\
\hline
\end{tabular}


Chroicocephalus cirrocephalus

Larus dominicanus

\section{Sternidae}

Sternula superciliaris

Phaetusa simplex

\section{Rynchopidae}

Rynchops niger

\section{Columbidae}

Columbina talpacoti

Columbina picui

Columba livia

Patagioenas picazuro

Zenaida auriculata

Leptotila verreauxi

Leptotila rufaxilla

Geotrygon montana

Psittacidae

Myiopsitta monachus

$\begin{array}{lllllllllll}\mathrm{x} & \mathrm{x} & \mathrm{x} & \mathrm{x} & \mathrm{x} & \mathrm{x} & \mathrm{x} & \mathrm{x} & \mathrm{x} & \mathrm{x} & \mathrm{x} \\ \mathrm{x} & \mathrm{x} & \mathrm{x} & \mathrm{x} & \mathrm{x} & \mathrm{x} & \mathrm{x} & \mathrm{x} & \mathrm{x} & \mathrm{x} & \mathrm{x} \\ \mathrm{x} & \mathrm{x} & & \mathrm{x} & & \mathrm{x} & \mathrm{x} & \mathrm{x} & \mathrm{x} & \mathrm{x} & \mathrm{x} \\ & & & & \mathrm{x} & & & & \mathrm{x} & & \\ & & & & \mathrm{x} & \mathrm{x} & \mathrm{x} & & \mathrm{x} & \mathrm{x} & \mathrm{x} \\ \mathrm{x} & \mathrm{x} & \mathrm{x} & \mathrm{x} & \mathrm{x} & & & \mathrm{x} & \mathrm{x} & & \mathrm{x} \\ & \mathrm{x} & & \mathrm{x} & & & & & & & \\ & \mathrm{x} & \mathrm{x} & & & & & & & & \end{array}$

92,86

Cuculidae

Micrococcyx cinereus

Piaya cayana

Coccyzus melacoryphus

$\mathrm{x}$

$\mathrm{x}$

$\mathrm{X}$

$\mathrm{x}$

$\mathrm{X}$

$\begin{array}{lllll}\mathrm{X} & \mathrm{X} & \mathrm{X} & \mathrm{X} & \mathrm{X}\end{array}$

85,71

Coccyzus americanus

Crotophaga ani

Guira guira

Tapera naevia

$\begin{array}{cccccc} & & \mathrm{x} & & & \\ \mathrm{x} & \mathrm{x} & \mathrm{x} & \mathrm{x} & \mathrm{x} & \mathrm{x} \\ \mathrm{x} & \mathrm{x} & \mathrm{x} & \mathrm{x} & & \\ & & \mathrm{x} & & & \\ \mathrm{x} & \mathrm{x} & \mathrm{x} & \mathrm{x} & \mathrm{x} & \mathrm{x} \\ \mathrm{x} & \mathrm{x} & \mathrm{x} & \mathrm{x} & \mathrm{x} & \mathrm{x} \\ & \mathrm{x} & \mathrm{x} & & & \end{array}$

$\mathrm{x}$

\section{Tytonidae}

Tyto alba

X $\mathrm{X}$

$\mathrm{X}$

$\mathrm{X}$

$\mathrm{x}$

28,57

\section{Strigidae}

Megascops choliba $\mathrm{x}$ $\mathrm{x}$

Megascops sanctaecatarinae

$\mathrm{x}$

$\mathrm{X}$

(x)

$\begin{array}{lll}\mathrm{x} & \mathrm{x} & \mathrm{x}\end{array}$

$\mathrm{X}$

$\mathrm{x}$

Athene cunicularia

$\mathrm{x}$ $\mathrm{X}$

Rhinoptynx clamator

\section{Caprimulgidae}

Chordeiles minor $\mathrm{x}$

Podager nacunda

$\mathrm{x}$

Nyctidromus albicollis

Caprimulgus longirostris X $\quad \mathrm{X}$

Caprimulgus parvulus

Hydropsalis torquata

$\mathrm{X}$ $\mathrm{x}$

\section{Apodidae}

Cypseloides fumigatus

Streptoprocne zonaris

$\mathrm{x} \quad \mathrm{x}$

$\mathrm{x}$

21,43 
Chaetura cinereiventris

Chaetura meridionalis

$\begin{array}{lllllllllllllll}\mathrm{X} & \mathrm{X} & \mathrm{X} & \mathrm{X} & \mathrm{X} & \mathrm{x} & \mathrm{x} & \mathrm{X} & \mathrm{x} & \mathrm{X} & \mathrm{X} & \mathrm{x} & \mathrm{x} & \mathrm{x} & 100\end{array}$

\section{Trochilidae}

\section{Florisuga fusca}

Anthracothorax nigricollis

Stephanoxis lalandi

Chlorostilbon lucidus

Thalurania glaucopis

Hylocharis chrysura

Leucochloris albicollis

Amazilia versicolor

\begin{tabular}{|c|c|c|c|c|c|c|c|c|c|c|c|c|c|c|}
\hline $\mathrm{x}$ & $\mathrm{x}$ & $\mathrm{x}$ & & $\mathrm{X}$ & & & & $\mathrm{X}$ & $\mathrm{X}$ & & & & & 42,86 \\
\hline \multirow[t]{2}{*}{$\mathrm{x}$} & $\mathrm{x}$ & $\mathrm{x}$ & & & $\mathrm{x}$ & & & $\mathrm{x}$ & $\mathrm{x}$ & & & & $\mathrm{X}$ & 50,00 \\
\hline & $\mathrm{x}$ & $\mathrm{x}$ & & & & & & $\mathrm{x}$ & & & & & & 21,43 \\
\hline \multirow[t]{2}{*}{$\mathrm{x}$} & $\mathrm{x}$ & $\mathrm{x}$ & $\mathrm{x}$ & & $\mathrm{x}$ & $\mathrm{X}$ & & $\mathrm{x}$ & $\mathrm{x}$ & & $\mathrm{x}$ & & $\mathrm{x}$ & 71,43 \\
\hline & $\mathrm{X}$ & $\mathrm{x}$ & & $X$ & & & & & & & & & & 21,43 \\
\hline $\mathrm{x}$ & $\mathrm{x}$ & $\mathrm{x}$ & $\mathrm{x}$ & $\mathrm{X}$ & $\mathrm{x}$ & $\mathrm{X}$ & $\mathrm{X}$ & $\mathrm{X}$ & $\mathrm{X}$ & $\mathrm{X}$ & $\mathrm{X}$ & $\mathrm{X}$ & $\mathrm{X}$ & 100 \\
\hline \multirow[t]{2}{*}{$x$} & $\mathrm{X}$ & $\mathrm{x}$ & $\mathrm{X}$ & & & & & $\mathrm{X}$ & & & & & & 35,71 \\
\hline & $\mathrm{x}$ & $\mathrm{x}$ & & & & & & & & & & & & 14,29 \\
\hline
\end{tabular}

\section{Alcedinidae}

Megaceryle torquata

$\begin{array}{llll}\mathrm{X} & \mathrm{x} & \mathrm{X} & \mathrm{x}\end{array}$

$\begin{array}{llll}\mathrm{X} & \mathrm{X} & \mathrm{X} & \mathrm{X}\end{array}$

Chloroceryle amazona

$\mathrm{x} \quad \mathrm{x}$

Chloroceryle americana

$\begin{array}{lll}\mathrm{X} & \mathrm{X}\end{array}$

$\begin{array}{lllll}\mathrm{X} & \mathrm{X} & \mathrm{X} & \mathrm{X} & \mathrm{X}\end{array}$

64,29

\section{Picidae}

Melanerpes candidus

$\mathrm{x} \quad \mathrm{x}$

$\mathrm{X}$

35,71

Veniliornis spilogaster

$\begin{array}{lllll}\mathrm{X} & \mathrm{X} & \mathrm{X} & \mathrm{X} & \mathrm{X}\end{array}$

14,29

Piculus aurulentus

Colaptes melanochloros

Colaptes campestris

Celeus flavescens

57,14

Dryocopus lineatus

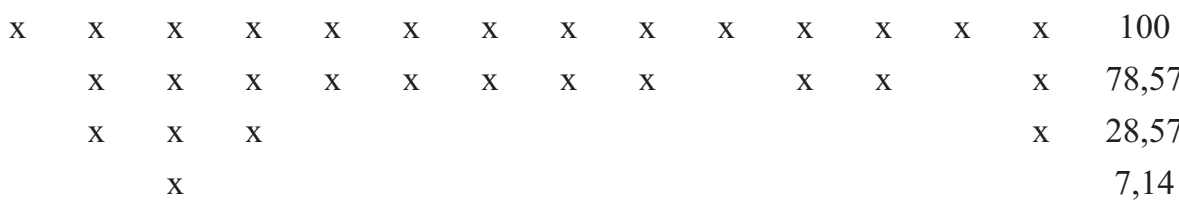

\section{Thamnophilidae}

Mackenziaena leachii

$\mathrm{X}$

X 14,29

Thamnophilus ruficapillus

Thamnophilus caerulescens

$\begin{array}{llll}\mathrm{X} & \mathrm{X} & \mathrm{X}\end{array}$

$\mathrm{x} \quad \mathrm{X} \quad \mathrm{x} \quad \mathrm{X}$

$\begin{array}{cccccc}X & X & X & X & & X \\ & X & & X & X & X\end{array}$

x 71,43

Conopophagidae

Conopophaga lineata

X $\quad \mathrm{X}$

$\mathrm{X}$

x 64,29

\section{Dendrocolaptidae}

Sittasomus griseicapillus

Lepidocolaptes falcinellus

x $\quad 7,14$

\section{Furnariidae}

Furnarius rufus

Schoeniophylax phryganophilus

Synallaxis ruficapilla

Synallaxis cinerascens

Synallaxis spixi

Cranioleuca obsoleta

Certhiaxis cinnamomeus

Anumbius annumbi

Syndactyla rufosuperciliata

Lochmias nematura

\begin{tabular}{|c|c|c|c|c|c|c|c|c|c|c|c|c|c|c|}
\hline \multirow[t]{3}{*}{$\mathrm{x}$} & $\mathrm{x}$ & $\mathrm{x}$ & $\mathrm{x}$ & $\mathrm{x}$ & $\mathrm{x}$ & $\mathrm{x}$ & $\mathrm{X}$ & $\mathrm{x}$ & $\mathrm{X}$ & $X$ & $\mathrm{x}$ & $\mathrm{x}$ & $\mathrm{x}$ & 100 \\
\hline & $\mathrm{x}$ & $\mathrm{x}$ & $\mathrm{x}$ & & & $\mathrm{x}$ & & $\mathrm{x}$ & & $\mathrm{x}$ & $\mathrm{x}$ & & & 50 \\
\hline & & & $\mathrm{x}$ & & & & & & & $\mathrm{X}$ & & & $\mathrm{X}$ & 21,43 \\
\hline$x$ & $\mathrm{x}$ & $\mathrm{x}$ & $\mathrm{x}$ & & & & & & & & & & $\mathrm{x}$ & 35,71 \\
\hline $\mathrm{x}$ & $\mathrm{x}$ & $\mathrm{x}$ & $\mathrm{x}$ & $\mathrm{x}$ & & & $\mathrm{x}$ & $\mathrm{x}$ & & $\mathrm{x}$ & & & $\mathrm{x}$ & 64,29 \\
\hline \multirow[t]{5}{*}{$\mathrm{X}$} & & & $\mathrm{x}$ & $\mathrm{x}$ & & $\mathrm{x}$ & & $\mathrm{x}$ & $\mathrm{X}$ & $\mathrm{X}$ & & & & 50 \\
\hline & $\mathrm{X}$ & $\mathrm{x}$ & $\mathrm{x}$ & & & $\mathrm{X}$ & & & & $\mathrm{X}$ & $\mathrm{x}$ & & & 42,86 \\
\hline & & & $\mathrm{x}$ & & $\mathrm{x}$ & $\mathrm{x}$ & & & & & $\mathrm{x}$ & & & 28,57 \\
\hline & & $\mathrm{x}$ & & & & $\mathrm{X}$ & $\mathrm{X}$ & & $\mathrm{X}$ & $\mathrm{X}$ & $\mathrm{x}$ & & $\mathrm{X}$ & 50 \\
\hline & $\mathrm{X}$ & $\mathrm{x}$ & & & & & & & & & & & & 14,29 \\
\hline
\end{tabular}




\begin{tabular}{|c|c|c|c|c|c|c|c|c|c|c|c|c|c|c|c|}
\hline \multicolumn{16}{|l|}{ Tyrannidae } \\
\hline Poecilotriccus plumbeiceps & & $\mathrm{x}$ & $\mathrm{x}$ & & & $\mathrm{x}$ & $\mathrm{x}$ & & $\mathrm{x}$ & & $\mathrm{x}$ & & & $\mathrm{x}$ & 50 \\
\hline Elaenia flavogaster & $\mathrm{x}$ & $\mathrm{x}$ & & $\mathrm{x}$ & $\mathrm{x}$ & & & $\mathrm{x}$ & $\mathrm{x}$ & $\mathrm{x}$ & $\mathrm{x}$ & $\mathrm{x}$ & & & 64,29 \\
\hline Elaenia spectabilis & & & & & & & & $\mathrm{x}$ & & & & & & & 7,14 \\
\hline Elaenia parvirostris & $\mathrm{x}$ & $\mathrm{x}$ & & $\mathrm{x}$ & $\mathrm{x}$ & & & $\mathrm{x}$ & $\mathrm{x}$ & $\mathrm{x}$ & & & & & 50 \\
\hline Elaenia mesoleuca & $\mathrm{x}$ & $\mathrm{x}$ & & & & & & & $\mathrm{x}$ & & & & & & 21,43 \\
\hline Elaenia obscura & $\mathrm{x}$ & $\mathrm{x}$ & & & & & & & $\mathrm{x}$ & & & & & & 21,43 \\
\hline Camptostoma obsoletum & $\mathrm{x}$ & $\mathrm{x}$ & $\mathrm{x}$ & $\mathrm{x}$ & $\mathrm{x}$ & $\mathrm{x}$ & & $\mathrm{x}$ & $\mathrm{x}$ & $\mathrm{x}$ & $\mathrm{x}$ & $\mathrm{x}$ & & $\mathrm{x}$ & 85,71 \\
\hline Serpophaga nigricans & & & & & & & & & & & & $\mathrm{x}$ & & & 7,14 \\
\hline Serpophaga subcristata & $\mathrm{x}$ & $\mathrm{x}$ & $\mathrm{x}$ & $\mathrm{x}$ & $\mathrm{x}$ & $\mathrm{x}$ & $\mathrm{x}$ & $\mathrm{x}$ & $\mathrm{x}$ & $\mathrm{x}$ & $\mathrm{x}$ & $\mathrm{x}$ & $\mathrm{x}$ & $\mathrm{x}$ & 100 \\
\hline Phylloscartes ventralis & & & & $\mathrm{x}$ & $\mathrm{x}$ & & & & & & $\mathrm{x}$ & & & $\mathrm{x}$ & 28,57 \\
\hline Tolmomyias sulphurescens & $\mathrm{x}$ & $\mathrm{x}$ & $\mathrm{x}$ & & & & & & $\mathrm{x}$ & & & & & & 28,57 \\
\hline Platyrinchus mystaceus & $\mathrm{x}$ & $\mathrm{x}$ & & & & & & & & & & & & & 14,29 \\
\hline Myiophobus fasciatus & $\mathrm{x}$ & $\mathrm{x}$ & $\mathrm{x}$ & $\mathrm{x}$ & & $\mathrm{x}$ & & & $\mathrm{x}$ & & $\mathrm{x}$ & & & & 50 \\
\hline Lathrotriccus euleri & $\mathrm{x}$ & $\mathrm{x}$ & & & $\mathrm{x}$ & & & & & & $\mathrm{x}$ & & & $\mathrm{x}$ & 35,71 \\
\hline Pyrocephalus rubinus & & & & & & & & & $\mathrm{x}$ & & & $\mathrm{x}$ & & & 14,29 \\
\hline Satrapa icterophrys & $\mathrm{x}$ & $\mathrm{x}$ & $\mathrm{x}$ & $\mathrm{x}$ & $\mathrm{x}$ & $\mathrm{x}$ & $\mathrm{x}$ & $\mathrm{x}$ & $\mathrm{x}$ & $\mathrm{x}$ & $\mathrm{x}$ & $\mathrm{x}$ & $\mathrm{x}$ & $\mathrm{x}$ & 100 \\
\hline Xolmis irupero & & $\mathrm{x}$ & & $\mathrm{x}$ & & $\mathrm{x}$ & $\mathrm{x}$ & & $\mathrm{x}$ & & & $\mathrm{x}$ & & & 42,86 \\
\hline Arundinicola leucocephala & & & $\mathrm{x}$ & & & & & & $\mathrm{x}$ & & & & & & 14,29 \\
\hline Machetornis rixosa & $\mathrm{x}$ & $\mathrm{x}$ & $\mathrm{x}$ & $\mathrm{x}$ & $\mathrm{x}$ & $\mathrm{x}$ & $\mathrm{x}$ & $\mathrm{x}$ & $\mathrm{x}$ & $\mathrm{x}$ & $\mathrm{x}$ & $\mathrm{x}$ & $\mathrm{x}$ & $\mathrm{x}$ & 100 \\
\hline Pitangus sulphuratus & $\mathrm{x}$ & $\mathrm{x}$ & $\mathrm{x}$ & $\mathrm{x}$ & $\mathrm{x}$ & $\mathrm{x}$ & $\mathrm{x}$ & $\mathrm{x}$ & $\mathrm{x}$ & $\mathrm{x}$ & $\mathrm{x}$ & $\mathrm{x}$ & $\mathrm{x}$ & $\mathrm{x}$ & 100 \\
\hline Myiodynastes maculatus & $\mathrm{x}$ & $\mathrm{x}$ & $\mathrm{x}$ & & $\mathrm{x}$ & & & & & & & & & & 28,57 \\
\hline Megarynchus pitangua & & $\mathrm{x}$ & & $\mathrm{x}$ & $\mathrm{x}$ & & & & & & & & & & 21,43 \\
\hline Empidonomus varius & $\mathrm{x}$ & $\mathrm{x}$ & $\mathrm{x}$ & & $\mathrm{x}$ & & & & & & & & & $\mathrm{x}$ & 35,71 \\
\hline Tyrannus melancholicus & $\mathrm{x}$ & $\mathrm{x}$ & $\mathrm{x}$ & $\mathrm{x}$ & $\mathrm{x}$ & $\mathrm{x}$ & $\mathrm{x}$ & $\mathrm{x}$ & $\mathrm{x}$ & $\mathrm{x}$ & $\mathrm{x}$ & $\mathrm{x}$ & $\mathrm{x}$ & $\mathrm{x}$ & 100 \\
\hline Tyrannus savana & $\mathrm{x}$ & $\mathrm{x}$ & $\mathrm{x}$ & $\mathrm{x}$ & $\mathrm{x}$ & $\mathrm{x}$ & $\mathrm{x}$ & $\mathrm{x}$ & $\mathrm{x}$ & $\mathrm{x}$ & $\mathrm{x}$ & $\mathrm{x}$ & $\mathrm{x}$ & & 92,86 \\
\hline Myiarchus swainsoni & $\mathrm{x}$ & $\mathrm{x}$ & $\mathrm{x}$ & $\mathrm{x}$ & $\mathrm{x}$ & & & & $\mathrm{x}$ & & & & & $\mathrm{x}$ & 50 \\
\hline
\end{tabular}

\begin{tabular}{lr} 
Pipridae & \\
Chiroxiphia caudata & $\mathrm{x}$ \\
\hline
\end{tabular}

Chiroxiphia caudata

$x$

\begin{tabular}{|c|c|c|c|c|c|c|c|c|c|c|c|}
\hline \multicolumn{12}{|l|}{ Tityridae } \\
\hline Schiffornis virescens & $\mathrm{x}$ & & & & & & & & & & 7,14 \\
\hline Pachyramphus polychopterus & $\mathrm{x}$ & $\mathrm{x}$ & $\mathrm{x}$ & $\mathrm{x}$ & & & & & & $\mathrm{x}$ & 35,71 \\
\hline \multicolumn{12}{|l|}{ Vireonidae } \\
\hline Cyclarhis gujanensis & $\mathrm{x}$ & $\mathrm{x}$ & $\mathrm{x}$ & $\mathrm{x}$ & $\mathrm{x}$ & $\mathrm{x}$ & $\mathrm{x}$ & $\mathrm{x}$ & $\mathrm{x}$ & $\mathrm{x}$ & 71,43 \\
\hline Vireo olivaceus & $\mathrm{x}$ & $\mathrm{x}$ & $\mathrm{x}$ & & & & $\mathrm{x}$ & $\mathrm{x}$ & & $\mathrm{x}$ & 42,86 \\
\hline
\end{tabular}

\section{Corvidae}

Cyanocorax caeruleus $\mathrm{X}$

\section{Hirundinidae}

Pygochelidon cyanoleuca Alopochelidon fucata

Stelgidopteryx ruficollis

Progne tapera

Progne chalybea

\begin{tabular}{|c|c|c|c|c|c|c|c|c|c|c|c|c|c|c|}
\hline $\mathrm{X}$ & $\mathrm{x}$ & $\mathrm{x}$ & $\mathrm{x}$ & $\mathrm{X}$ & $\mathrm{x}$ & $\mathrm{x}$ & $\mathrm{X}$ & $\mathrm{x}$ & $\mathrm{x}$ & $\mathrm{X}$ & $\mathrm{x}$ & $\mathrm{x}$ & $\mathrm{x}$ & 100 \\
\hline $\mathrm{X}$ & $\mathrm{x}$ & & $\mathrm{x}$ & & & & & $\mathrm{X}$ & & & & & & 28,57 \\
\hline $\mathrm{X}$ & $\mathrm{x}$ & $\mathrm{x}$ & & & & & & $\mathrm{x}$ & & & & & & 28,57 \\
\hline $\mathrm{x}$ & $\mathrm{x}$ & $\mathrm{x}$ & $\mathrm{x}$ & $\mathrm{X}$ & $\mathrm{x}$ & $\mathrm{x}$ & & $\mathrm{x}$ & $\mathrm{x}$ & $\mathrm{X}$ & $\mathrm{x}$ & $\mathrm{x}$ & & 85,71 \\
\hline $\mathrm{X}$ & $\mathrm{x}$ & $\mathrm{X}$ & $\mathrm{X}$ & $\mathrm{X}$ & & & & $\mathrm{x}$ & $\mathrm{X}$ & & & & & 50 \\
\hline
\end{tabular}


Tachycineta leucorrhoa

$\begin{array}{llll}\mathrm{X} & \mathrm{x} & \mathrm{x}\end{array}$

$\mathrm{X} \quad \mathrm{X}$

$\mathrm{x} \quad \mathrm{x}$

57,14

Hirundo rustica

$\mathrm{X}$

Troglodytidae

Troglodytes musculus

X X

Polioptilidae

Polioptila dumicola

x $\quad \mathrm{X} \quad \mathrm{x}$

$\mathrm{x} x$

57,14

\section{Turdidae}

Turdus rufiventris

Turdus leucomelas

Turdus amaurochalinus

Turdus subalaris

X $\mathrm{X}$

X $\quad \mathrm{X}$

100

Turdus albicollis

$\begin{array}{cccccc} & & & & \mathrm{x} & \\ \mathrm{X} & \mathrm{X} & \mathrm{X} & \mathrm{x} & \mathrm{x} & \mathrm{x} \\ \mathrm{X} & \mathrm{X} & \mathrm{X} & \mathrm{x} & \mathrm{X} & \\ \mathrm{X} & \mathrm{X} & \mathrm{X} & & & \end{array}$

$\mathrm{x} x$

$\mathrm{x}$

X

\section{Mimidae}

Mimus saturninus

$\mathrm{X}$

$\mathrm{X}$

X

X 28,57

Mimus triurus

X

\section{Motacillidae}

Anthus lutescens

X

14,29

\section{Coerebidae}

Coereba flaveola

$\mathrm{X} \quad \mathrm{X}$

\section{Thraupidae}

Pyrrhocoma ruficeps

Trichothraupis melanops

Tachyphonus coronatus

Thraupis sayaca

Thraupis palmarum

Thraupis bonariensis

Stephanophorus diadematus

Pipraeidea melanonota

Tangara preciosa

$\begin{array}{llll} & \mathrm{X} & & \\ \mathrm{X} & \mathrm{X} & & \mathrm{X} \\ \mathrm{X} & \mathrm{X} & \mathrm{X} & \mathrm{X}\end{array}$

21,43

Tersina viridis

X $x \quad x$

100

Hemithraupis guira

\section{Emberizidae}

Zonotrichia capensis Ammodramus humeralis

Haplospiza unicolor

Donacospiza albifrons

Poospiza nigrorufa

Poospiza cabanisi

Sicalis flaveola

Sicalis luteola

Embernagra platensis Volatinia jacarina Sporophila collaris

\begin{tabular}{|c|c|c|c|c|c|c|c|c|c|c|c|c|c|c|}
\hline \multirow[t]{6}{*}{$x$} & $\mathrm{x}$ & $\mathrm{x}$ & $\mathrm{x}$ & $\mathrm{x}$ & $\mathrm{x}$ & $\mathrm{x}$ & $\mathrm{x}$ & $\mathrm{x}$ & $\mathrm{x}$ & $\mathrm{x}$ & $\mathrm{x}$ & $\mathrm{x}$ & $\mathrm{x}$ & 100 \\
\hline & $\mathrm{x}$ & & $\mathrm{x}$ & & & & & & $\mathrm{x}$ & & & & & 21,43 \\
\hline & $\mathrm{x}$ & & & & & & & & & & & & & 7,14 \\
\hline & $\mathrm{x}$ & & $\mathrm{x}$ & & & & & $\mathrm{x}$ & & & & & & 21,43 \\
\hline & $\mathrm{x}$ & $\mathrm{x}$ & $\mathrm{x}$ & & & $\mathrm{x}$ & & $\mathrm{x}$ & $\mathrm{x}$ & $\mathrm{x}$ & & & $\mathrm{x}$ & 57,14 \\
\hline & $\mathrm{x}$ & & & & & & $\mathrm{x}$ & $\mathrm{x}$ & & $\mathrm{x}$ & & & $\mathrm{x}$ & 35,71 \\
\hline \multirow[t]{3}{*}{$\mathrm{x}$} & $\mathrm{x}$ & $\mathrm{x}$ & $\mathrm{x}$ & $\mathrm{x}$ & $\mathrm{x}$ & $\mathrm{x}$ & $\mathrm{X}$ & $\mathrm{x}$ & $\mathrm{x}$ & $\mathrm{x}$ & $\mathrm{x}$ & $\mathrm{x}$ & & 92,86 \\
\hline & $\mathrm{x}$ & & $\mathrm{x}$ & & & $\mathrm{x}$ & & & & & $\mathrm{x}$ & & & 28,57 \\
\hline & & & $\mathrm{x}$ & & & & & & & & & & & 7,14 \\
\hline $\mathrm{x}$ & $\mathrm{x}$ & $\mathrm{x}$ & $\mathrm{x}$ & $\mathrm{x}$ & & $\mathrm{x}$ & & & & & $\mathrm{x}$ & & & 50 \\
\hline $\mathrm{x}$ & & & & & & & & & & & & & & 7,14 \\
\hline
\end{tabular}


Sporophila caerulescens

Coryphospingus cucullatus

$$
\begin{array}{lllll}
\mathrm{X} & \mathrm{X} & \mathrm{x} & \mathrm{X} & \mathrm{x}
\end{array}
$$

Paroaria coronata

$\begin{array}{lllllll}\mathrm{x} & \mathrm{x} & \mathrm{x} & \mathrm{x} & \mathrm{x} & & \\ & \mathrm{x} & & & \mathrm{x} & \mathrm{x}\end{array}$

$\mathrm{x} \quad \mathrm{x} \quad \mathrm{x}$

$\mathrm{x}$

64,29

Cardinalidae

Saltator similis

$\mathrm{X}$

$\mathrm{x}$

$\mathrm{x} \quad \mathrm{X}$

42,86

Cyanoloxia brissonii

Cyanoloxia glaucocaerulea

21,43

14,29

\section{Parulidae}

Parula pitiayumi

Geothlypis aequinoctialis

Basileuterus culicivorus

Basileuterus leucoblepharus

\section{Icteridae}

Icterus cayanensis

$\mathrm{x} \quad \mathrm{X}$

$\mathrm{X} \quad \mathrm{X}$

Chrysomus ruficapillus

Pseudoleistes guirahuro

Agelaioides badius

Molothrus rufoaxillaris

Molothrus bonariensis

Sturnella superciliaris

$\begin{array}{llll}\mathrm{X} & \mathrm{X} & \mathrm{X} & \mathrm{X} \\ \mathrm{X} & \mathrm{X} & \mathrm{X} & \mathrm{X} \\ \mathrm{X} & \mathrm{X} & \mathrm{X} & \mathrm{X} \\ \mathrm{X} & \mathrm{X} & \mathrm{X} & \mathrm{X}\end{array}$

$\begin{array}{ll}\mathrm{X} & \mathrm{x} \\ \mathrm{x} & \mathrm{x} \\ \mathrm{X} & \mathrm{x} \\ & \mathrm{x}\end{array}$

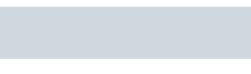

$\begin{array}{cccccc} & & & & & \\ \mathrm{X} & \mathrm{X} & \mathrm{X} & \mathrm{X} & \mathrm{X} & 100 \\ \mathrm{X} & \mathrm{X} & \mathrm{X} & & \mathrm{X} & 85,71 \\ \mathrm{X} & \mathrm{X} & & & \mathrm{X} & 85,71 \\ \mathrm{X} & \mathrm{X} & & & \mathrm{X} & 78,57\end{array}$

\begin{tabular}{|c|c|c|c|c|c|c|c|c|c|c|c|c|c|c|}
\hline & $\mathrm{x}$ & $\mathrm{x}$ & $\mathrm{X}$ & $\mathrm{X}$ & & & & $\mathrm{x}$ & $\mathrm{X}$ & & & & & 42,86 \\
\hline \multirow[t]{2}{*}{$\mathrm{x}$} & $\mathrm{X}$ & $\mathrm{X}$ & $\mathrm{X}$ & & $X$ & $\mathrm{X}$ & & $\mathrm{X}$ & & $\mathrm{x}$ & $\mathrm{X}$ & & & 64,29 \\
\hline & & & $\mathrm{X}$ & & & & & & & & & & & 7,14 \\
\hline \multirow[t]{2}{*}{$\mathrm{x}$} & $\mathrm{X}$ & $\mathrm{X}$ & & $\mathrm{X}$ & $\mathrm{X}$ & $\mathrm{X}$ & $\mathrm{X}$ & $\mathrm{X}$ & $\mathrm{X}$ & & $\mathrm{X}$ & & & 71,43 \\
\hline & & & & & & & & $\mathrm{X}$ & & & & & & 7,14 \\
\hline \multirow[t]{3}{*}{$\mathrm{x}$} & $\mathrm{X}$ & $\mathrm{X}$ & $\mathrm{X}$ & $\mathrm{X}$ & $\mathrm{X}$ & $\mathrm{X}$ & $\mathrm{X}$ & $\mathrm{X}$ & $\mathrm{X}$ & $\mathrm{X}$ & $\mathrm{X}$ & $\mathrm{X}$ & $\mathrm{X}$ & 100 \\
\hline & & & $\mathrm{X}$ & & & & & $\mathrm{X}$ & & & $\mathrm{X}$ & & & 21,43 \\
\hline & & $\mathrm{X}$ & & & & & & $\mathrm{X}$ & $\mathrm{X}$ & & & & & 21,43 \\
\hline$X$ & $\mathrm{X}$ & $\mathrm{X}$ & $\mathrm{X}$ & $\mathrm{X}$ & $\mathrm{X}$ & & $\mathrm{X}$ & $\mathrm{X}$ & $\mathrm{X}$ & & & $\mathrm{X}$ & & 71,43 \\
\hline $\mathrm{X}$ & $\mathrm{X}$ & & & & & & & $\mathrm{X}$ & & & & & & 21,43 \\
\hline $\mathrm{X}$ & $\mathrm{X}$ & $\mathrm{X}$ & & & & & $\mathrm{X}$ & $\mathrm{X}$ & $\mathrm{X}$ & & & & & 42,86 \\
\hline
\end{tabular}

\section{Fringillidae}

Carduelis magellanica

Euphonia chlorotica

Euphonia violacea

Euphonia cyanocephala

$x \quad x \quad x$

$\begin{array}{llll} & \mathrm{X} & \mathrm{X} & \mathrm{X}\end{array}$

$\mathrm{X} \quad \mathrm{X}$

$\begin{array}{lllll}X & X & X & X\end{array}$

$\mathrm{X} \quad \mathrm{X}$

64,29

Passeridae

Passer domesticus

$\begin{array}{lllllll}\mathrm{X} & \mathrm{X} & \mathrm{X} & \mathrm{X} & \mathrm{X} & \mathrm{X}\end{array}$

$\begin{array}{llllllllllllll}96 & 149 & 142 & 123 & 71 & 74 & 82 & 62 & 126 & 72 & 72 & 84 & 39 & 75\end{array}$

Total

A análise de similaridade de Jaccard (Figura 2) agrupou as áreas verdes localizadas em Porto Alegre em um conjunto distinto daquelas localizadas na região metropolitana da capital gaúcha (exceto o Jardim Botânico de Porto Alegre). É provável que tal distinção esteja relacionada à heterogeneidade de ambientes e ao hábitat matriz em que as áreas estão inseridas. As áreas verdes localizadas na região metropolitana de Porto Alegre, como o Parque Municipal Getúlio Vargas, o campus da Universidade do Vale do Rio dos Sinos e o Parque Zoológico de Sapucaia do Sul, estão inseridas em um hábitat matriz mais heterogêneo, que inclui edificações e construções urbanas, e áreas úmidas de entorno (como arrozais e maricazais). Já as áreas verdes de Porto Alegre estão inseridas em um hábitat matriz basicamente urbano. O Jardim Botânico de Porto Alegre, apesar de não possuir área expressiva, se comparado a outros locais, apresenta grande heterogeneidade ambiental, possibilitando a ocorrência de diversas espécies também encontradas nas áreas verdes da região metropolitana. O Parque Gabriel Knijnik foi o que mais se diferenciou quanto à composição de espécies. Nesta área, a vegetação nativa é bem desenvolvida, há presença de muitas árvores frutíferas e o parque é adjacente a uma 


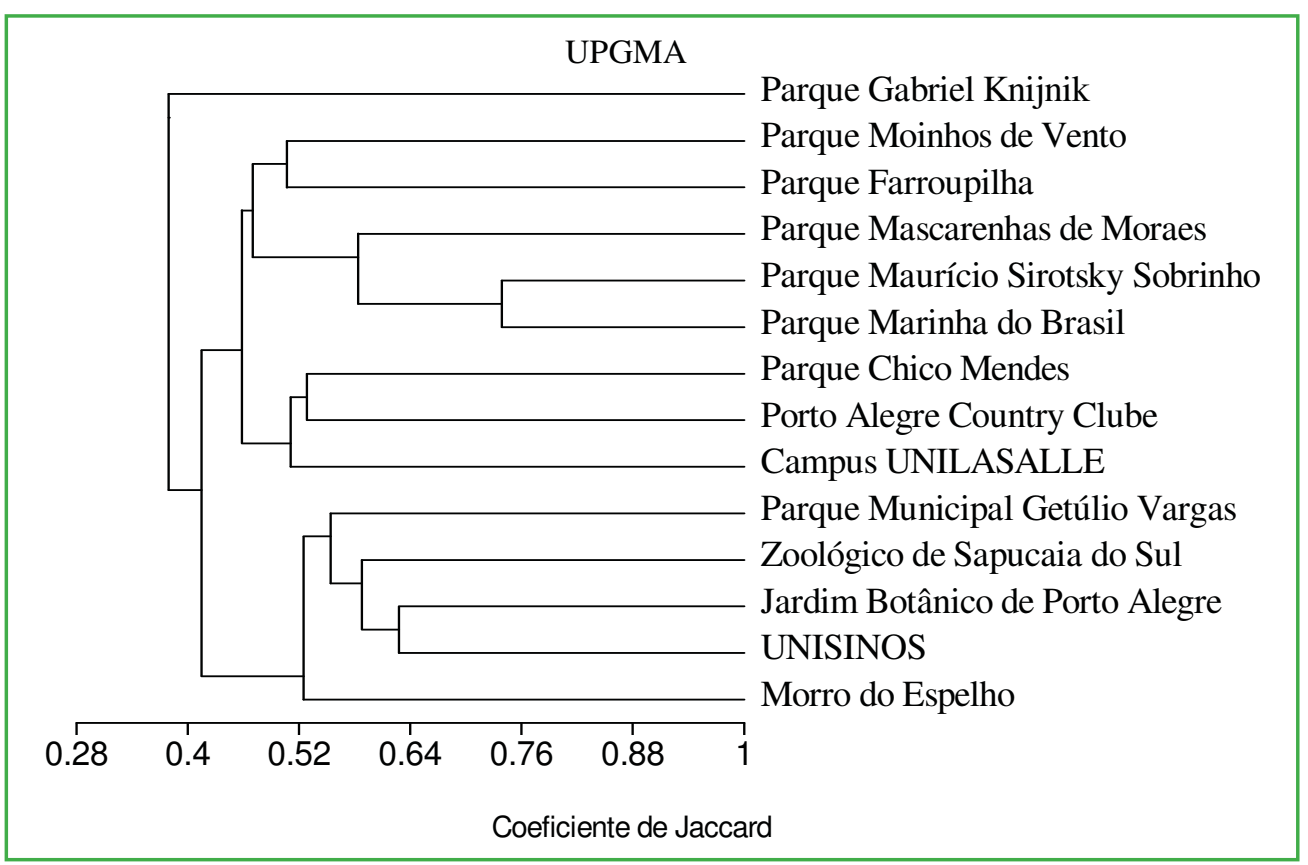

FIGURA 2: Análise de similaridade de Jaccard comparando as áreas verdes localizadas na região metropolitana de Porto Alegre quanto à composição da avifauna.

área com densa cobertura vegetal nativa (Efe et al., 2001; Menegat, 2006). Esses fatos, provavelmente, favorecem a ocorrência de espécies associadas a ambientes florestais, como Crypturellus obsoletus, Piculus aurulentus e Lepidocolaptes falcinellus, e de frugívoros mais especializados como, por exemplo, Ortalis guttata e Chiroxiphia caudata. Um aspecto importante a ser considerado refere-se à diferença quanto aos esforços amostrais despendidos em cada área. Em algumas áreas verdes mais de um levantamento foi realizado, o que faz com que a informação referente à avifauna seja mais completa do que em áreas que apresentam apenas um levantamento.

\section{Padrões de distribuição das espécies de aves}

O padrão resultante da compilação de dados demonstrou que 110 espécies possuem distribuição restrita a determinadas áreas verdes da região metropolitana de Porto Alegre. Algumas dessas espécies limitam-se a determinados tipos de hábitats, tais como ambientes florestais, a exemplo de C. obsoletus, O. guttata, Celeus flavescens, L. falcinellus, Synallaxis ruficapilla, Lochmias nematura, C. caudata e Pyrrhocoma ruficeps (Sick,
1997; Belton, 1994). Outras espécies são características de áreas abertas úmidas, como Amazonetta brasiliensis, Ixobrychus involucris, Pardirallus nigricans e Sturnella superciliaris, ou de áreas abertas secas, como Nothura maculosa e Anumbius annumbi, por exemplo (Sick, 1997; Belton, 1994). Muitas das espécies incluídas nesta categoria também se mostraram ocasionais em algumas áreas, ou seja, foram registradas em poucas ocasiões durante os levantamentos de campo conduzidos por cada pesquisador, tais como Sarkidiornis sylvicola, Nyctanassa violacea, Percnohierax leucorrhous e Dryocopus lineatus no P. Z. de Sapucaia do Sul (Voss, 1977c), Chordeiles minor, Schiffornis virescens e Sporophila collaris no Morro do Espelho (Tampson, 1990), e Anhinga anhinga, Ictinia plumbea e Tersina viridis no campus da Universidade do Vale do Rio dos Sinos (Grillo e Bencke, 1995).

Quarenta e cinco espécies ocorreram entre 35,7\% e $57,1 \%$ das áreas verdes, apresentando-se parcialmente distribuídas na região metropolitana de Porto Alegre. Apesar da maioria dessas espécies ser comum em ambientes urbanos (Belton, 1994), suas ocorrências estão condicionadas à existência de algum ambiente particular. Este é o caso de Gallinula chloropus, Certhiaxis cinnamomeus e Poospiza nigrorufa, as quais 
são características de áreas úmidas com vegetação associada, tais como banhados (Belton, 1994), sendo encontradas apenas na presença de tais ambientes.

Sessenta e uma espécies são amplamente distribuídas em áreas verdes urbanas da região metropolitana de Porto Alegre, ocorrendo em mais de 64\% das áreas analisadas. Tais espécies podem ser consideradas mais generalistas quanto à escolha de habitats (Sick, 1997; Willis, 2000), a exemplo de Rupornis magnirostris, Crotophaga ani, Guira guira, Hylocharis chrysura, Camptostoma obsoletum, Turdus amaurochalinus, Thraupis sayaca, T. bonariensis, Sicalis flaveola e Euphonia chlorotica.

Tendo em conta a lista de espécies resultante da revisão da literatura, cabe destacar que vários dos trabalhos consultados são antigos e que provavelmente algumas espécies de aves se extinguiram localmente em virtude das alterações ambientais ocorridas nas áreas ao longo dos anos. Grillo e Bencke (1995) comentam que muitas espécies desapareceram da área do campus da Unisinos em decorrência de alterações físicas resultantes de obras de ampliação de infra-estrutura. Os autores mencionam que espécies associadas a ambientes úmidos, como Laterallus melanophaius, Gallinula melanops, $C$. cinnamomeus e Ammodramus humeralis, desapareceram devido ao aterramento de áreas alagadas e banhados, enquanto que a diminuição das áreas de mata resultou no desaparecimento de Stephanoxis lalandi, Trichothraupis melanops e Haplospiza unicolor. Já Stephanophorus diadematus e Cyanoloxia brissonii desapareceram, provavelmente, devido à captura para o comércio ilegal de animais silvestres (Grillo e Bencke, 1995).

\section{Análise das áreas verdes quanto à heterogeneidade de habitats e à rique-}

\section{za de espécies de aves}

A análise de regressão múltipla demonstrou que a área total, o número de hábitats presentes e a área do vizinho mais próximo influenciam positivamente a riqueza de espécies de aves nas áreas verdes urbanas $\left(\mathrm{R}^{2}=0,63 ; \mathrm{F}=5,734 ; \mathrm{gl}=3,10 ; \mathrm{P}=0,015\right)$. Este resultado era esperado, uma vez que áreas de maiores dimensões (Parque Municipal Getúlio Vargas, Campus da Universidade do Vale do Rio dos Sinos e Parque
Zoológico de Sapucaia do Sul) possuem maior heterogeneidade de hábitats, tais como capões de vegetação nativa, lagos, bosques e áreas de campo, o que favorece a ocorrência de táxons adaptados aos diferentes ambientes, incrementando a riqueza de espécies (Anjos e Laroca, 1989; Matarazzo-Neuberguer, 1995; Krügel e Anjos, 2000; Lopes e Anjos, 2006). Outras áreas, como o Jardim Botânico de Porto Alegre, o Parque Gabriel Knijnik e o Morro do Espelho, apesar de não apresentarem grandes dimensões, reúnem distintos ambientes, aspecto que contribui para a ocorrência de muitas aves. A área do vizinho mais próximo também é um importante fator a ser considerado. Áreas de maiores dimensões possuem alta riqueza de espécies e podem atuar como fontes de indivíduos a áreas adjacentes mais próximas (Gotelli, 2009). O método stepwise excluiu a distância do vizinho mais próximo como fator significativo para explicar a variação na riqueza de espécies de aves entre as áreas verdes urbanas.

Os resultados obtidos a partir da revisão da literatura e da análise de características físicas das áreas verdes avaliadas sugerem que a composição e a riqueza de espécies de aves, nessas áreas da região metropolitana de Porto Alegre, podem estar relacionadas com o tamanho e com a heterogeneidade ambiental de cada local. Áreas verdes de maiores dimensões e aquelas com maior variedade de hábitats comportaram maior riqueza de espécies de aves. Este cenário reforça a importância, para a conservação da biodiversidade em médio e longo prazos, de estudos que busquem esclarecer os padrões de distribuição das aves em ecossistemas urbanos. Uma questão relevante a ser respondida é se pequenas praças e ruas arborizadas constituem corredores ecológicos para as espécies de aves que ocorrem em áreas verdes urbanas.

Outra questão fundamental à análise da comunidade de aves em ambientes urbanos refere-se à obtenção de dados de frequência. Não se pode caracterizar a avifauna dessas áreas com base em espécies raras ou registros ocasionais, mas sim em espécies com registros constantes em cada área (Fontana, 2005). Alguns dos trabalhos consultados não apresentam resultados de frequência de ocorrência das espécies, dado imprescindível à distinção de táxons raros e mais comuns, o que impossibilitou a 
definição de critérios mais rigorosos para a inclusão das espécies de aves nas análises. Assim, sugere-se que os estudos sobre avifauna contemplem, pelo menos, uma análise de frequência de ocorrência, de modo a subsidiar tal caracterização.

Os resultados apresentados neste estudo não devem ser tomados como definitivos, uma vez que podem ter sido influenciados por esforços amostrais desiguais entre as áreas, diferentes observadores e, principalmente, por aspectos temporais. Além disso, o caráter extremamente dinâmico destes ecossistemas faz com que muitas espécies venham a ser registradas em locais onde antes não ocorriam. Em algumas ocasiões, M.F.B.S (primeiro autor deste artigo) registrou novas ocorrências para algumas áreas verdes, a citar, Tolmomyias sulphurescens, Pachyramphus viridis e Trichothraupis melanops no P. M. Getúlio Vargas, em Canoas.

O conhecimento acumulado sobre a diversidade da avifauna da região metropolitana de Porto Alegre, na forma de publicações e listas de espécies, demonstrou ser bastante rico e profícuo, permitindo inferir que fatores físicos correlacionados (tamanho da área, heterogeneidade de hábitats) são componentes importantes a serem considerados na análise da comunidade de aves em áreas urbanizadas e em estratégias de conservação.

\section{Agradecimentos}

Agradecemos aos dois revisores que contribuíram expressivamente com críticas e sugestões para o aprimoramento deste estudo. Ao amigo Rafael Gomes de Moura, pela confecção do mapa.

\section{Referências}

Accordi, I. A. 2001. Avifauna de três sítios contíguos na zona urbana do município de Canoas, RS. Acta Biologica Leopoldensia, 23 (1): 59-68.

Aleixo, A. 2001. Conservação da avifauna da Floresta Atlântica: Efeitos da fragmentação e a importância de florestas secundárias. In: Albuquerque, J. L. B.; Cândido Jr., J. F.; Straube, F. C. \& Roos, A. L (Eds). Ornitologia e conservação: Da ciência às estratégias. Unisul, Tubarão, Brasil, p.199-206.
Anjos, L. 1990. Distribuição de aves em uma floresta de araucária da cidade de Curitiba (Sul do Brasil). Acta Biológica Paranaense, 19 (4): 51-63.

Anjos, L.; Laroca, S. 1989. Abundância relativa e diversidade específica de aves em duas comunidades urbanas de aves de Curitiba (Sul do Brasil). Brazilian Archives of Biology and Technology, 32 (4): 637-643.

Belton, W. 1994. Aves do Rio Grande do Sul: Distribuição e biologia. Unisinos, São Leopoldo, Brasil, 584pp.

Bencke, G. A. 2001. Lista de referência das aves do Rio Grande do Sul. Fundação Zoobotânica do Rio Grande do Sul, Porto Alegre, Brasil, 104pp.

Bencke, G. A.; Fontana C. S.; Dias, R. A.; Maurício, G. N.; Mahler Jr, J. K. F. 2003. Aves. In: Fontana C. S.; Bencke, G. A. \& Reis, R. E. (Eds). Livro vermelho da fauna ameaçada de extinção no Rio Grande do Sul. EDIPUCRS, Porto Alegre, Brasil, p.189-479.

Comitê Brasileiro de Registros Ornitológicos. 2008. Listas das aves do Brasil. Versão 05/10/2008. Disponível em <http://www. cbro.org.br>. Acesso em 16 abril 2009.

Datasinos. 2006. Projeto Datasinos. Sistema de Informação e Análise Sócio-Econômico-Ambiental para o COREDE do vale do Rio dos Sinos. Disponível em: $<$ http://www.datasinos.unisinos. $\mathrm{br} /$ consulta138.htm $>$. Acesso em 26 de agosto de 2009

Efe, M. A.; Mohr, L. V.; Bugoni, L. 2001. Guia ilustrado das aves dos parques de Porto Alegre. PROAVES, SMAM, COPESUL, CEMAVE, Porto Alegre, Brasil, 144pp.

Fontana, C. S. 2005. A ornitofauna em Porto Alegre no século XX: Status de ocorrência e conservação. Comunicações do Museu de Ciências - PUCRS, Série Zoologia, 18 (2): 73-212.

Fundação Zoobotânica do Rio Grande do Sul - Jardim Botânico de Porto Alegre. 2004. Plano Diretor do Jardim Botânico de Porto Alegre. Publicações Avulsas FZB, Porto Alegre, Brasil 100pp.

Gimenes, R. G.; Anjos, L. 2003. Efeitos da fragmentação florestal sobre as comunidades de aves. Acta Scientiarum, Biological Sciences, 25 (2): 391-402.

Grillo, H. C. Z.; Bencke, G. A. 1995. Aves do novo campus da Universidade do Vale do Rio dos Sinos, UNISINOS, São Leopoldo, RS. Acta Biologica Leopoldensia, 17 (1): 123-145.

Gotelli, N. J. 2009. Ecologia. Editora Planta. 287 pp.

Kovach, W. L. 2005. MVSP - A multivariate statistical package for Windows, ver. 3.1. Kovach Computing Services, Pentraeth, Wales, UK.

Krügel, M. M.; Anjos, L. 2000. Bird communities in forest remnants in the city of Maringá, Paraná State, Southern Brazil. Ornitologia Neotropical 11: $315-330$

Lopes, E. V.; Anjos, L. 2006. A composição da avifauna do campus da Universidade Estadual de Londrina, norte do Paraná, Brasil. Revista Brasileira de Zoologia 23 (1): 145-156

Matarazzo-Neuberguer, W. M. 1995. Comunidades de aves de cinco parques e praças da Grande São Paulo, Estado de São Paulo. Ararajuba, 3 : 13-19.

Mendonça-Lima, A.; Fontana, C. S. 2000. Composição, freqüência e aspectos biológicos da avifauna no Porto Alegre Country Clube, Rio Grande do Sul. Ararajuba, 8 (1): 1-8.

Menegat, R. (Coord.). 2006. Atlas ambiental de Porto Alegre. 3. ed., rev. Porto Alegre: Ed. da UFRGS, 228pp 
Oliveira, K. F. de. 2006. Lista comentada da avifauna do campus Unilasalle - Canoas, RS. Trabalho de Conclusão de Curso de Graduação, Centro Universitário La Salle, Brasil, 66pp.

Sander, M.; Voss W. A. 1982. Aves livres observadas no Parque Farroupilha, Porto Alegre, Rio Grande do Sul, Brasil. Pesquisas Série Zoologia, 33: 3-16.

Secretaria de Planejamento e Gestão. 2008. Atlas socioeconômico do Rio Grande do Sul. Disponível em <http://www.scp.rs.gov.br/ atlas/atlas.asp?menu=298>. Acesso em 10 julho de 2008.

Sick, H. 1997. Ornitologia brasileira. Nova Fronteira, Rio de Janeiro, Brasil, 714pp.

SMAM. 2009. Secretaria Municipal do Meio Ambiente de Porto Alegre. Disponível em <http://www2.portoalegre.rs.gov.br/ smam/\#> Acesso em 21 de agosto de 2009.

Tampson, V. E. 1990. Lista comentada das espécies de aves registradas para o Morro do Espelho, São Leopoldo, Rio Grande do Sul, Brasil (1983-1988). Acta Biologica Leopoldensia, 12 (1): 19-37.

Valadão, R. M.; Franchin, A. G.; Marçal Júnior, O. 2006. A avifauna no Parque Municipal Victório Siquierolli, zona urbana de Uberlândia (MG). Biotemas, 19 (1): 81-91

Voss, W. A. 1976. Aves observadas nas cidades de Novo Hamburgo e São Leopoldo, RS. Estudos Leopoldenses, 36: 43-53.
Voss, W. A. 1977a. Aves de São Leopoldo. I - Aves observadas no Bairro Recreio. Estudos Leopoldenses, 41: 36-39.

Voss, W. A. 1977b. Aves de São Leopoldo. II - Aves observadas no banhado da Olaria Linck. Estudos Leopoldenses, 41: 41-46.

Voss, W. A. 1977c. Aves silvestres livres observadas no Parque Zoológico de Sapucaia do Sul, RS, Brasil e outros ensaios. Pesquisas, 30: 1-29.

Voss, W. A. 1979. Aves observadas na área central da cidade de São Leopoldo, RS. Pesquisas, 31: 9-24.

Voss, W. A. 1981. Aves de Porto Alegre, Rio Grande do Sul. III Aves observadas no Jardim Botânico da Fundação Zoobotânica do Rio Grande do Sul. Acta Biologica Leopoldensia, 3 (1): 81-94.

Voss, W. A.; Sander, M. 1979a. Aves de São Leopoldo. III - Aves observadas no Centro de Recreação do Trabalhador. Estudos Leopoldenses, 50: 71-77.

Voss, W. A.; Sander, M. 1979b. Aves de São Leopoldo. IV - Aves observadas no Novo Campus da Universidade do Vale do Rio dos Sinos - UNISINOS. Estudos Leopoldenses, 50: 79-83.

Willis, E. O. 2000. Ranking urban avifaunas (Aves) by number of localities per species in São Paulo, Brazil. Iheringia, 88: 139-146.

Wilkinson, L. 2004. Systat 11. Systat Software Inc, San José, USA. 